\title{
Development of an integrated Sasang constitution diagnosis method using face, body shape, voice, and questionnaire information
}

\author{
Jun-Hyeong Do ${ }^{1 \dagger}$, Eunsu Jang ${ }^{1 \dagger}$, Boncho Ku${ }^{1}$, Jun-Su Jang ${ }^{1}$, Honggie Kim² and Jong Yeol Kim ${ }^{{ }^{*}}$
}

\begin{abstract}
Background: Sasang constitutional medicine (SCM) is a unique form of traditional Korean medicine that divides human beings into four constitutional types (Tae-Yang: TY, Tae-Eum: TE, So-Yang: SY, and So-Eum: SE), which differ in inherited characteristics, such as external appearance, personality traits, susceptibility to particular diseases, drug responses, and equilibrium among internal organ functions. According to SCM, herbs that belong to a certain constitution cannot be used in patients with other constitutions; otherwise, this practice may result in no effect or in an adverse effect. Thus, the diagnosis of SC type is the most crucial step in SCM practice. The diagnosis, however, tends to be subjective due to a lack of quantitative standards for SC diagnosis.

Methods: We have attempted to make the diagnosis method as objective as possible by basing it on an analysis of quantitative data from various Oriental medical clinics. Four individual diagnostic models were developed with multinomial logistic regression based on face, body shape, voice, and questionnaire responses. Inspired by SCM practitioners' holistic diagnostic processes, an integrated diagnostic model was then proposed by combining the four individual models.

Results: The diagnostic accuracies in the test set, after the four individual models had been integrated into a single model, improved to $64.0 \%$ and $55.2 \%$ in the male and female patient groups, respectively. Using a cut-off value for the integrated SC score, such as 1.6, the accuracies increased by $14.7 \%$ in male patients and by $4.6 \%$ in female patients, which showed that a higher integrated SC score corresponded to a higher diagnostic accuracy.

Conclusions: This study represents the first trial of integrating the objectification of SC diagnosis based on quantitative data and SCM practitioners' holistic diagnostic processes. Although the diagnostic accuracy was not great, it is noted that the proposed diagnostic model represents common rules among practitioners who have various points of view. Our results are expected to contribute as a desirable research guide for objective diagnosis in traditional medicine, as well as to contribute to the precise diagnosis of SC types in an objective manner in clinical practice.
\end{abstract}

Keywords: Sasang constitution, Diagnosis, Face, Body shape, Voice, Questionnaire, Integration

\footnotetext{
* Correspondence: ssmed@kiom.re.kr

${ }^{\dagger}$ Equal contributors

${ }^{1}$ Constitutional Medicine \& Diagnosis Research Group, Medical Research

Division, Korea Institute of Oriental Medicine, 1672 Yuseongdae-ro,

Yuseong-gu, Daejeon 305-811, Republic of Korea

Full list of author information is available at the end of the article
} 


\section{Background}

Sasang constitutional medicine (SCM) is a unique form of traditional Korean medicine that divides human beings into four constitutional types (Tae-Yang: TY, TaeEum: TE, So-Yang: SY, and So-Eum: SE), which differ in inherited characteristics, such as external appearance, personality traits, susceptibility to particular diseases, drug responses, and equilibrium among internal organ functions [1]. In contrast to the diagnostic procedure of traditional Chinese medicine, which places the greatest importance on the 'syndrome,' SCM places emphasis on the 'constitution,' and the therapeutic decision is then mostly based on which SC type the patient is [2]. According to SCM, each constitutional type corresponds to a certain group of medicinal herbs and herbal remedies. This relationship means that herbs that belong to a certain constitution cannot be used in patients with other constitutions; otherwise, this practice may result in no effect or in an adverse effect. Thus, the diagnosis of SC type is the most crucial step in SCM practice [3].

In agreement with other forms of complementary and alternative medicine [4-7], the diagnostic process in SCM remains subjective and unreliable due to a lack of quantitative standards for SC diagnosis [8]. So far, attempts at standardization have focused on facial, body shape, voice analyses, and questionnaires. In terms of facial analysis, facial metrics on 2D and 3D images have been employed to investigate the typical features of SC types and to develop predictive models for SC types [9-13]. In body shape analysis, five horizontal lines $[14,15]$ and eight circumferences [16,17] of the trunk are commonly used. Research on voice analysis has been undertaken regarding various vocal features, such as the pitch, frequency, reading speed, shimmer, harmonics, formants, and energy of the voice, using computerized speech laboratory methods [18-20]. In 2004, a voice analysis system named the Phonetic System for Sasang Constitution-2004 was successfully developed [21,22]. Questionnaires have been used in the earliest trials of SC diagnosis [23,24]. Today, the Questionnaire for Sasang Constitutional Classification II (QSCCII) is a commonly used questionnaire with qualitative components concerning face, body shape, voice, and personal characteristics, in addition to physiological symptoms [25].

Most of this research has focused on the construction of individual diagnostic models using data from a small number of clinical sites, which was insufficient to guarantee the validity of the diagnostic models. In clinical practice, SCM practitioners employ all four examinations to determine patients' SC types. This holistic approach provides better information about the patients' constitutions and ailments, which cannot be obtained by individual examination. An integrated diagnostic tool that simulates SCM practitioners' holistic diagnostic processes would be a more desirable approach.

In this study, four standardized individual diagnostic models were developed based on a large amount of data that were acquired from various Oriental medical clinics for the four diagnostic components: face, body shape, voice, and questionnaire responses. For a quantitative and objective analysis, engineering techniques and statistical data analysis including multinomial logistic regression were employed. Inspired by SCM practitioners' holistic diagnostic processes, an integrated diagnostic model was then proposed by combining the four individual models. The validity of the proposed model was confirmed by applying it to a test set that was not used in developing the model.

\section{Methods}

\section{Participants and data acquisition}

We strictly controlled the characteristics of the practitioners and subjects. 23 and more SCM practitioners, who had more than five years of experience in clinical practice, diagnosed the patients' SC types. Their SC types were confirmed by observing improvements after the administration of constitution-specific pharmaceuticals over one month. A more detailed procedure of determining SC type is described in Song et al. [26].

We collected face, body shape, voice, and questionnaire data from the subjects using a standard operating procedure that was developed for the Korea Constitutional Multicenter Study [27]. The collected data included photographs with neutral expressions from the frontal and profile points of view; height, weight, and eight body shape circumferences; voice signal recordings of five vowels ('a,' 'e, 'i,' 'o,' and 'u') and two repeated sentences; and questionnaires for yin/yang characteristics and physiological symptoms. This process was approved by the Korea Institute of Oriental Medicine (KIOM) Institutional Review Board (I-0910/02-001) and we obtained written informed consent from the subjects.

From 23 sites (Oriental medical clinics), 2,973 patients, ranging in age from teenagers to people in their eighties, were recruited between November of 2007 and July of 2011 (see Additional file 1). Among these 2,973 patients, 2,462 patients recruited between November of 2007 and July of 2010 were assigned to training set for the construction of a diagnostic model, whereas the others were assigned to test set for the validation of the constructed model. All data that contained clinical information were stored in the Korea Constitutional Multicenter Bank at KIOM.

Several patients were excluded for various reasons. TY-type subjects were unavoidably excluded due to the small sample size of the TY type. Subjects younger than 15 years of age were also excluded on the basis that the phenotypic characteristics of these subjects would 
greatly fluctuate based on age during the adolescent period. And improper data were excluded from each component. Table 1 summarizes the excluded data and the number of used data for the training and test of each model after the exclusion.

\section{Candidate feature variables \\ Facial images}

Candidate feature variables expressing facial characteristics were created with facial points and contours, which were automatically extracted via image processing techniques. The positions of the numbered facial points and features are shown in Additional file 2, and the candidate feature variables are described in Additional file 3.

\section{Body shape}

The eight circumferences of body shape, ratios of all possible pairs of eight circumferences, height, weight, and body mass index (BMI) were used as candidate variables expressing body shape characteristics. The measurement methods of the circumferences are described in Additional file 4, where a standardized tapeline $(150 \mathrm{~cm} /$ 60 inches, Hoechstmass, Germany) was used to measure body shape.

\section{Voice}

Voice features were extracted using two voice analysis programs, HTK [28] and Praat [29]. The size of a window, i.e., the minimum duration of a voice signal for feature extraction, was $40 \mathrm{~ms}$, and neighboring windows overlapped by $50 \%$. We used 41 features for each vowel and 17 features for the sentences. The descriptions of vowel and sentence features are shown in Additional file 5 and 6 , respectively. A total of 222 features were extracted as an initial feature set. Next, we applied a genetic algorithm-based feature selection technique using Weka [30] to reduce the total number of features. Finally, 88 features were selected and used in diagnostic model learning.

\section{Questionnaire}

Binary variables representing personality characteristics and physiological symptoms were constructed using the response categories of the questions in the questionnaire, which consisted of 67 multiple-choice questions (see Additional file 7). Because face, body shape, and voice features were all represented by continuous variables, efforts were made to generate continuous variables using the binary variables that were obtained with the questionnaire so as to apply the same analytic method used for the other features. The procedure of generating continuous variables is summarized in Additional file 8 [31]. Selected significant binary variables are also shown in Additional files 9, 10, 11, 12, 13 and 14.

\section{Compensating for age differences}

Because the candidate feature variables may have shown age-specific trends, a process to eliminate the effect of age was considered. To estimate the non-linear trend of each feature variable according to age, moving averages and standard deviations of the variables at each age were calculated using the samples within an age range of \pm 5 years for the given age. In calculating the moving average, outliers were excluded based on the results of the multivariate outlier detection method [32,33]. Each candidate feature variable was then normalized with the average and the standard deviation of the variable.

Table 1 The numbers of excluded and used data for the training and test of each individual diagnostic model

\begin{tabular}{|c|c|c|c|c|c|c|c|c|}
\hline \multirow{2}{*}{$\begin{array}{l}\text { List of exclusion reasons (Total no. of } \\
\text { subjects: training 2462; test } 511 \text { ) }\end{array}$} & \multicolumn{4}{|c|}{ Training } & \multicolumn{4}{|c|}{ Test } \\
\hline & Face & $\begin{array}{l}\text { Body } \\
\text { shape }\end{array}$ & Voice & Questionnaire & Face & $\begin{array}{l}\text { Body } \\
\text { shape }\end{array}$ & Voice & Questionnaire \\
\hline Initially not collected & 22 & 0 & 409 & 409 & 0 & 0 & 0 & 0 \\
\hline Low quality pictures or voice recording files & 133 & - & 132 & - & 6 & - & 14 & - \\
\hline TY samples & 58 & 59 & 54 & 56 & 6 & 6 & 6 & 6 \\
\hline Subjects younger than 15 years old & 52 & 54 & 39 & 41 & 13 & 13 & 11 & 13 \\
\hline Data extraction errors and missing cases & 1189 & 97 & 3 & 0 & 132 & 0 & 0 & 0 \\
\hline $\begin{array}{l}\text { Outliers considered in the calculation of } \\
\text { the moving average }\end{array}$ & 46 & 27 & 86 & 16 & 0 & 7 & 26 & 0 \\
\hline Excluding influential samples & 6 & 11 & 49 & 25 & 0 & 0 & 0 & 0 \\
\hline Final no. of subjects & 956 & 2214 & 1687 & 1915 & 354 & 485 & 480 & 492 \\
\hline $\begin{array}{l}\text { Common samples after } \\
\text { integrating the four components } \\
\text { (for the integrated diagnostic model) }\end{array}$ & \multicolumn{4}{|c|}{729 (male: 241, female: 488) } & \multicolumn{4}{|c|}{346 (male: 114, female: 232) } \\
\hline
\end{tabular}




\section{Model for Sasang constitution diagnosis Individual diagnostic models}

Before building the individual diagnostic model for each component, unusual samples were excluded that influenced the predicted estimates. Two binary logistic regression analyses that compared TE to SE and SE to SY were conducted, and the unusually influential samples were then identified by calculating Cook's distance and standard residuals [34]. The excluded data are provided in Table 1.

With the data remaining after excluding the influential samples, individual diagnostic models were developed by multinomial logistic regression (MLR) based on stepwise forward variable selection using the score statistic and Wald's test [35,36]. Each individual diagnostic model consisted of two independent models: one was for male patients and the other for female patients. The age factor was forced into each model as a baseline covariate for the adjustment. For the diagnostic model that was generated using questionnaire information, the factors of age, education and occupation level, which may have affected the questionnaire responses, were used as baseline covariates.

\section{Integrating diagnostic models from four diagnostic components}

Let $\pi_{i j}$ be the estimated probability of the $i^{\text {th }}$ subject in category $j$ for each individual diagnostic model, where $j=1,2$, and 3 indicates the TE, SE, and SY types, respectively. The estimated probability can be regarded as a score for the different SC types, which we called SC score; hence, it was feasible to consider the total of each $\pi_{i j}$ derived from the four individual diagnostic models as the integrated score. In addition, the importance of each individual diagnostic model was also considered by multiplying the weights by $\pi_{i j}$. The integrated score of an SC type $j$ for the $i^{t h}$ subject, denoted as TSCORE $E_{i j}$, can be defined by the sum of $\left(\pi_{i j}\right)_{r}$ with weight $w_{r}$ :

$$
\operatorname{TSCORE}_{i j}=\sum_{r=1}^{4} w_{r}\left(\pi_{i j}\right)_{r}
$$

where $r$ indicates each individual diagnostic component; $r=1,2,3$, and 4 , representing face, body shape, voice, and questionnaire, respectively.

In this study, it was assumed that all individual diagnostic components equally contributed to the diagnosis of the SC type, and, hence, all weights for each individual SC score were initially set to 1 . Finally, the predicted SC type for the $i^{t h}$ subject was determined by choosing the maximum value between TSCORE $E_{i 1}, T S C O R E_{i 2}$, and $T S C O R E_{i 3}$. This process can be expressed as the following equation:

$$
\begin{aligned}
& \text { Predicted } S C_{i} \\
& \quad=\operatorname{argmax}\left(\operatorname{TSCORE}_{i 1}, \operatorname{TSCORE}_{i 2}, \operatorname{TSCORE}_{i 3}\right),
\end{aligned}
$$

where each number of the subscriptions indicates TE, SE, and SY. For better clarity, the schematic diagnosis algorithm is illustrated in Figure 1.

\section{Results}

Predicted results of the individual diagnostic models

The accuracies of the created individual diagnostic models for both the training and test sets are shown in Table 2, and the selected variables and estimated parameters that were created by the MLR are summarized in Additional files 15, 16, 17, 18, 19, 20, 21 and 22 .

The accuracies of the face, body shape, voice, and questionnaire models were $57.5 \%, 61.3 \%, 57.1 \%$, and

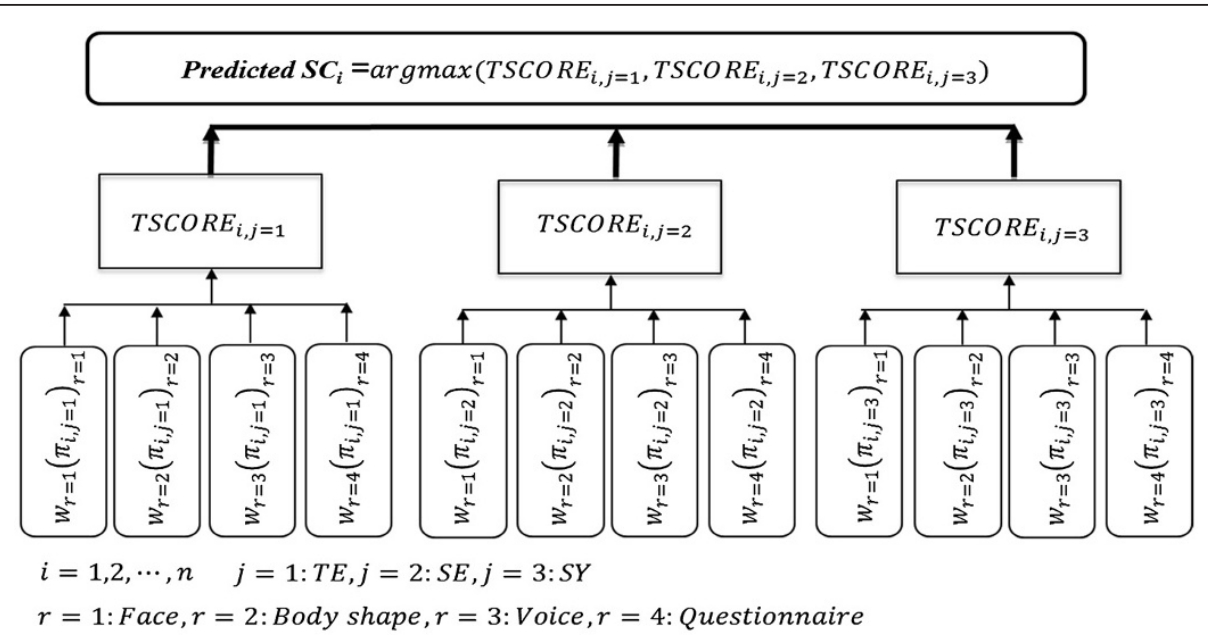

Figure 1 Schematic flow chart of the integration process using the probability scores from the four diagnostic components. 
Table 2 Accuracy of the individual diagnostic models

\begin{tabular}{|c|c|c|c|c|c|c|c|c|}
\hline & \multicolumn{4}{|c|}{ Male } & \multicolumn{4}{|c|}{ Female } \\
\hline & Face & $\begin{array}{l}\text { Body } \\
\text { shape }\end{array}$ & Voice & Questionnaire & Face & $\begin{array}{l}\text { Body } \\
\text { shape }\end{array}$ & Voice & Questionnaire \\
\hline Training Set & $57.5 \%$ & $61.3 \%$ & $57.1 \%$ & $57.4 \%$ & $55.6 \%$ & $54.2 \%$ & $46.1 \%$ & $53.8 \%$ \\
\hline Test Set & $60.5 \%$ & $54.8 \%$ & $39.9 \%$ & $57.7 \%$ & $42.9 \%$ & $60.2 \%$ & $37.5 \%$ & $43.2 \%$ \\
\hline
\end{tabular}

$57.4 \%$, respectively, in the training set and were $60.5 \%$, $54.8 \%, 39.9 \%$, and $57.7 \%$, respectively, in the test set for male patients. The accuracies of the face, body shape, voice, and questionnaire models were 55.6\%, 54.2\%, $46.1 \%$, and $53.8 \%$, respectively, in the training set and $42.9 \%, 60.2 \%, 37.5 \%$, and $43.2 \%$, respectively, in the test set for female patients.

The results indicate that the accuracies for male patients were higher than for female patients, except for the test results for body shape. In the diagnostic model for voice, the accuracy of the test set drastically decreased compared to that of the training set, which meant that the validity of the diagnostic model for voice was less than that of the other individual diagnostic models. In addition, the coefficient of determination $R^{2}$ for the model for voice was relatively lower than the others (see Additional files 15, 16, 17, 18, 19, 20, 21 and 22).

\section{Predicted results of the integrated diagnostic model}

The proposed integrated diagnostic model was tested with the lower weight for voice $\left(w_{3}=0.5\right)$, as well as with equal weights $\left(w_{\mathrm{r}}=1\right)$, to examine the effect of reducing the weight for voice due to its low validity. Both trials, wherein an equal or half weight was established for voice, are evaluated in Tables 3 and 4, respectively.

Overall, the results of the integrated model were superior to those of the individual models. The accuracy of the integrated model with equal weights was $69.3 \%$ for the training set and $61.4 \%$ for the test set in male patients and $63.5 \%$ for the training set and $52.2 \%$ for the test set in female patients. The accuracy of the integrated model, applying $w_{3}=0.5$, was $68.9 \%$ for the training set and $64.0 \%$ for the test set in male patients and $62.3 \%$ for the training set and $55.2 \%$ for the test set in female patients.

The result when applying $w_{3}=0.5$ was more accurate than when applying $w_{3}=1$ for the test set, whereas the accuracy of the model when applying $w_{3}=0.5$ was lower than when applying $w_{3}=1$ for the training set. This result indicated that the validity of the model when applying $w_{3}=0.5$ was higher than when applying $w_{3}=1$. The sensitivity of the model was relatively high for TE but was comparatively low for SY male and SE female.

Figure 2 depicts the accuracy of the integrated model according to the maximum value of the integrated SC scores, $\max \left(\operatorname{TSCORE}_{i 1}\right.$, TSCORE $\left._{i 2}, T_{S C O R E_{i 3}}\right)$. Accuracy showed an increasing trend as the maximum value increased. To enhance the accuracy of the diagnostic model, a cut-off value was selected to diagnose only those subjects whose maximum value of integrated SC scores was higher than the cut-off value. If a cut-off value of 1.6 was chosen, for example, then the accuracies of the integrated model were $76.3 \%$ and $70.4 \%$ for male and female patients, respectively, in the training set $\left(w_{3}=0.5\right)$ and $78.7 \%$ and $59.8 \%$ for male and female patients, respectively, in the test set $\left(w_{3}=0.5\right)$.

Table 3 Diagnostic results of the integrated diagnostic model with equal weights $\left(w_{r}=1\right)$

\begin{tabular}{|c|c|c|c|c|c|c|c|c|c|c|c|c|c|c|}
\hline & \multicolumn{7}{|c|}{ Male } & \multicolumn{7}{|c|}{ Female } \\
\hline & & & \multicolumn{3}{|c|}{ Predicted SC type } & \multirow[b]{2}{*}{ Total } & \multirow[b]{2}{*}{ Sensitivity } & & & \multicolumn{3}{|c|}{ Predicted SC type } & \multirow[b]{2}{*}{ Total } & \multirow[b]{2}{*}{ Sensitivity } \\
\hline & & & $\mathrm{TE}$ & SE & SY & & & & & $\mathrm{TE}$ & SE & SY & & \\
\hline \multirow[t]{6}{*}{ Training set } & True & TE & 87 & 2 & 7 & 96 & $90.6 \%$ & True & TE & 125 & 2 & 43 & 170 & $73.5 \%$ \\
\hline & SC & $\overline{\mathrm{SE}}$ & 14 & 38 & 11 & 63 & $60.3 \%$ & SC & SE & 16 & 50 & 60 & 126 & $39.7 \%$ \\
\hline & Type & $S Y$ & 29 & 11 & 42 & 82 & $51.2 \%$ & type & SY & 36 & 21 & 135 & 192 & $70.3 \%$ \\
\hline & & Total & 130 & 51 & 60 & 241 & & & $\overline{\text { Total }}$ & 177 & 73 & 238 & 488 & \\
\hline & $\overline{\text { Accur }}$ & & & & & & & Accur & & & & & & \\
\hline & & & $\mathrm{TE}$ & SE & SY & Total & Sensitivity & & & TE & SE & SY & Total & Sensitivity \\
\hline \multirow[t]{5}{*}{ Test set } & True & TE & 47 & 2 & 5 & 54 & $87.0 \%$ & True & TE & 55 & 4 & 35 & 94 & $58.5 \%$ \\
\hline & SC & $\overline{\mathrm{SE}}$ & 7 & 14 & 6 & 27 & $51.9 \%$ & SC & $\overline{\mathrm{SE}}$ & 5 & 27 & 39 & 71 & $38.0 \%$ \\
\hline & Type & $S Y$ & 16 & 8 & 9 & 33 & $27.3 \%$ & type & $S Y$ & 23 & 5 & 39 & 67 & $58.2 \%$ \\
\hline & & $\overline{\text { Total }}$ & 70 & 24 & 20 & 114 & & & Total & 83 & 36 & 113 & 232 & \\
\hline & $\overline{\text { Accur }}$ & & & & & & & Accur & & & & & & \\
\hline
\end{tabular}


Table 4 Diagnostic results of the integrated diagnostic model with the lower weight for voice $\left(w_{3}=0.5\right)$

\begin{tabular}{|c|c|c|c|c|c|c|c|c|c|c|c|c|c|c|}
\hline & \multicolumn{7}{|c|}{ Male } & \multicolumn{7}{|c|}{ Female } \\
\hline & & & \multicolumn{3}{|c|}{ Predicted SC type } & \multirow[b]{2}{*}{ Total } & \multirow[b]{2}{*}{ Sensitivity } & & & \multicolumn{3}{|c|}{ Predicted SC type } & \multirow[b]{2}{*}{ Total } & \multirow[b]{2}{*}{ Sensitivit } \\
\hline & & & $\overline{\mathrm{TE}}$ & SE & SY & & & & & $\overline{\mathrm{TE}}$ & SE & SY & & \\
\hline \multirow[t]{6}{*}{ Training set } & True & TE & 85 & 3 & 8 & 96 & $88.5 \%$ & True & TE & 122 & 2 & 46 & 170 & $71.8 \%$ \\
\hline & SC & $\overline{\mathrm{SE}}$ & 12 & 37 & 14 & 63 & $58.7 \%$ & $\mathrm{SC}$ & $\overline{S E}$ & 16 & 51 & 59 & 126 & $40.5 \%$ \\
\hline & Type & $\overline{S Y}$ & 27 & 11 & 44 & 82 & $53.7 \%$ & type & SY & 38 & 23 & 131 & 192 & $68.2 \%$ \\
\hline & & $\overline{\text { Total }}$ & 124 & 51 & 66 & 241 & & & Total & 176 & 76 & 236 & 488 & \\
\hline & $\overline{\text { Accur }}$ & & & & & & & Accur & & & & & & \\
\hline & & & TE & SE & SY & Total & Sensitivity & & & TE & SE & SY & Total & Sensitivity \\
\hline \multirow[t]{5}{*}{ Test set } & True & $\mathrm{TE}$ & 47 & 3 & 4 & 54 & $87.0 \%$ & True & $\mathrm{TE}$ & 55 & 5 & 34 & 94 & $58.5 \%$ \\
\hline & SC & $\overline{\mathrm{SE}}$ & 7 & 14 & 6 & 27 & $51.9 \%$ & SC & $\overline{\mathrm{SE}}$ & 3 & 31 & 37 & 71 & $43.7 \%$ \\
\hline & type & SY & 13 & 8 & 12 & 33 & $36.4 \%$ & type & $S Y$ & 21 & 4 & 42 & 67 & $62.7 \%$ \\
\hline & & $\overline{\text { Total }}$ & 67 & 25 & 22 & 114 & & & Total & 79 & 40 & 113 & 232 & \\
\hline & \multicolumn{3}{|c|}{$\overline{\text { Accuracy }}$} & & \multicolumn{2}{|c|}{$64.0 \%$} & & \multicolumn{2}{|c|}{ Accuracy } & \multicolumn{5}{|c|}{$55.2 \%$} \\
\hline
\end{tabular}

\section{Discussion \& conclusion}

In this study, attempts were made to design a diagnostic model that was as objective as possible based on an analysis of quantitative data from various Oriental medical clinics. We tried to extract common criteria for SC diagnosis from a great variety of data, including practitioners' varying points of view. Four individual diagnostic models for face, body shape, voice, and questionnaire information were obtained. Then, an integrated model was proposed that was inspired by SCM practitioners' holistic diagnostic processes, which provided more sufficient information and which could be a more desirable approach for SC diagnosis.

In the individual training set, the diagnostic accuracy for body shape in the group of male patients was as high as $61.3 \%$. The accuracies for face and questionnaire responses were almost the same, whereas that of voice was slightly lower. In the group of female patients, the diagnostic accuracy for face was $55.6 \%$, followed by body shape,

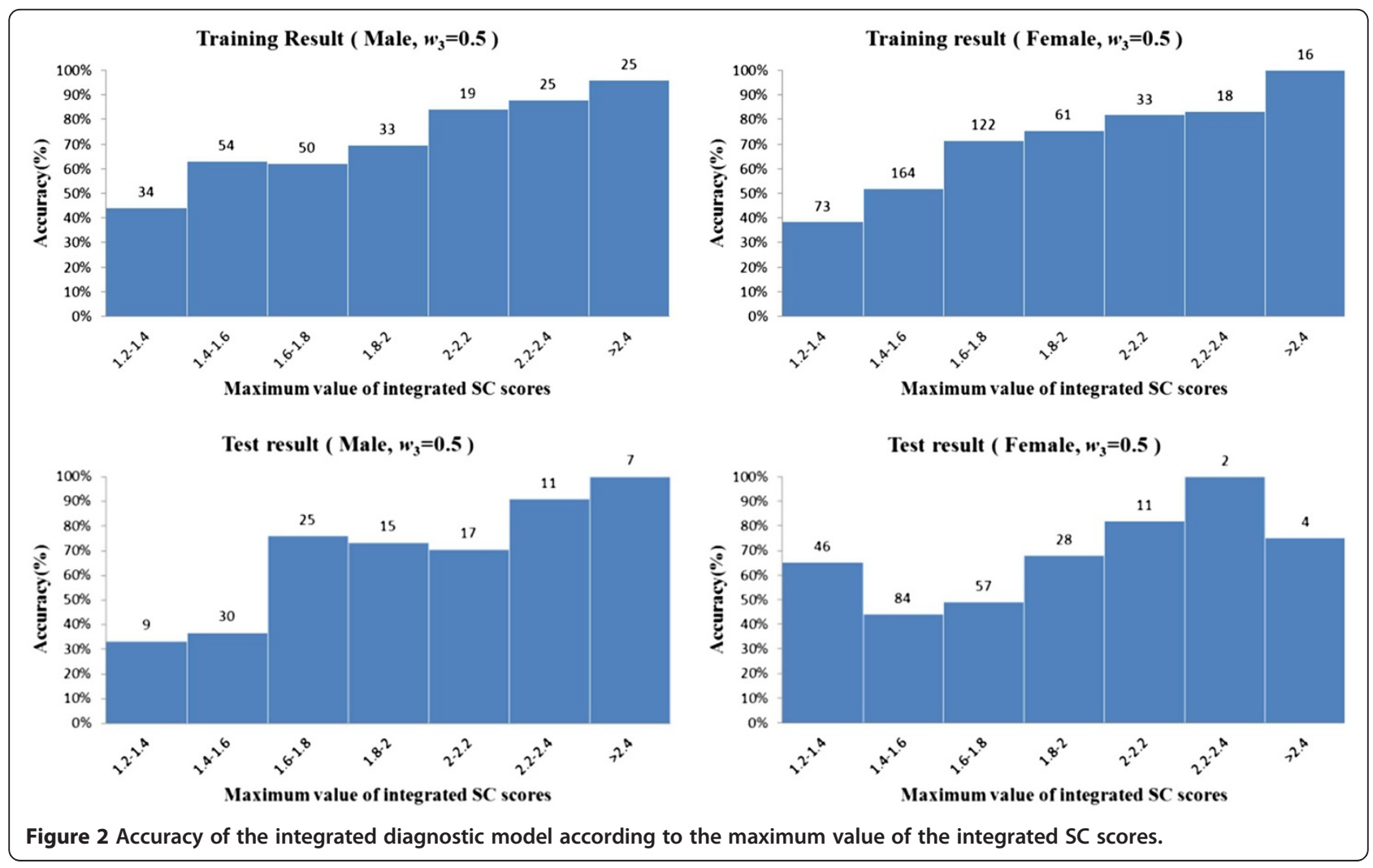


questionnaire, and voice, respectively, with almost an identical accuracy except for voice, as in the case of the male patients. The relatively low accuracy of the model for voice implies that it is difficult to find a stable diagnostic rule for voice. The individual diagnostic accuracies in the test set were slightly lower than those of the training set; however, the decrease in accuracy was within an acceptable range, except for the model for voice.

The diagnostic accuracies in the training set, after the four individual models had been integrated into a single model, improved to $69.3 \%$ and $63.5 \%$ in the male and female patient groups, respectively; however, when the model was applied to the test set, the accuracies decreased to $61.4 \%$ in the male patients and $52.2 \%$ in the female patients. The poor individual performance for voice was blamed, which led us to test an alternative integrated model by assigning a weight of half for voice. Although the new model showed almost the same diagnostic accuracy for the training set $(68.9 \%$ in male patients and $62.3 \%$ in female patients), it clearly produced a greater accuracy in the test set (64.0\% in male patients and $55.2 \%$ in female patients).

Although the resulting diagnostic power was not great, it was better than that of QSCCII [25], which is 51\% and has been widely used for the diagnosis of SC type [37]. It may be difficult to directly compare our new method to QSCCII because QSCCII was developed using only questionnaire information and was tested on limited data collected from a single site; however, an individual's response to qualitative questions on the four diagnostic components in the QSCCII could be subjective depending on his/her own point of view. It should be noted that the integrated model using quantitative data is superior in terms of validity and objectiveness.

A more desirable result was that a higher integrated SC score corresponded to a higher diagnostic accuracy, as shown in Figure 2. Using a cut-off value for the integrated SC score, such as 1.6, the accuracies increased by $14.7 \%$ in male patients and by $4.6 \%$ in female patients in the test set.

The insufficient diagnostic accuracies can be explained as follows. First, we collected data from 23 different sites, which incurred the possibility that the data might still contain too many practitioners' subjective opinions. This fact could have affected the SC diagnostic accuracy, although the characteristics of the practitioners and the subjects were kept very strict. Evidence of there being different opinions among practitioners can be found in other research [38,39].

Second, some of the variables that were extracted from face, body shape, voice, and questionnaire responses did not show clear differences among the SC types (see Additional files 9, 10, 11, 12, 13, 14, 15, 16, 17, 18, 19 and 20). This finding reveals that there still exists limitation to fully describing constitutional characteristics, as listed quantitatively in the SCM literature.
Third, because the Confucian culture of Korea has an influence on Korean women to modify their characteristics and their voices in talking, it is more difficult to obtain a diagnostic model in female patients than in male patients. The relatively low accuracies for questionnaire responses and voice partly explain this influence.

The proposed model was implemented in the form of a web-based prototype and is currently being tested in several clinics to get feedback from the practitioners. In the future, it will be necessary to collect more data on the TY type to complete the SC diagnostic model. Despite 2,973 samples being collected, only 1,075 samples were used, mainly due to a lack of featured extraction techniques and a lack of data quality control. It is necessary to develop an advanced technique for automatic feature extraction and to explore new feature variables for an improved diagnostic method. A different weighting method might be considered to properly reflect the importance of each diagnostic component. Furthermore, a future study may place emphasis on improving the performance for the groups which have poor diagnostic accuracies and sensitivities. At this point, we might need to analyze the typical subjects chosen by consensus from among SCM practitioners.

This study represents the first trial of integrating the objectification of SC diagnosis based on quantitative data and SCM practitioners' holistic diagnostic processes. Although the diagnostic accuracy was not great, it is noted that the proposed diagnostic model represents common rules among practitioners who have various points of view. Our results are expected to contribute as a desirable research guide for objective diagnosis in traditional medicine, as well as to contribute to the precise diagnosis of SC types in an objective manner in clinical practice.

\section{Additional files}

Additional file 1: Table S1. Population characteristics of the participants.

Additional file 2: Figure S1. Facial points used to calculated candidate feature variables.

Additional file 3: Table S2. Feature variables expressing the facial characteristics.

Additional file 4: Table S3. Measurement methods of eight circumferences for body shape.

Additional file 5: Table S4. Description of vowel features.

Additional file 6: Table S5. Description of sentence features. Additional file 7: Table S6. List of questions.

Additional file 8: Table S7. The procedure of generating continuous variables with the response categories of the questions in the questionnaire.

Additional file 9: Table S8. Significant binary variables of the questionnaire in TE male patients.

Additional file 10: Table S9. Significant binary variables of the questionnaire in SE male patients.

Additional file 11: Table S10. Significant binary variables of the questionnaire in SY male patients. 
Additional file 12: Table S11. Significant binary variables of the questionnaire in TE female patients.

Additional file 13: Table S12. Significant binary variables of the questionnaire in SE female patients.

Additional file 14: Table S13. Significant binary variables of the questionnaire in SY female patients.

Additional file 15: Table S14. Selected variables and estimated parameters for face (male).

Additional file 16: Table S15. Selected variables and estimated parameters for face (female).

Additional file 17: Table S16. Selected variables and estimated parameters for body shape (male).

Additional file 18: Table S17. Selected variables and estimated parameters for body shape (female).

Additional file 19: Table S18. Selected variables and estimated parameters for voice (male).

Additional file 20: Table S19. Selected variables and estimated parameters for voice (female).

Additional file 21: Table S20. Selected variables and estimated parameters for questionnaire (male).

Additional file 22: Table S21. Selected variables and estimated parameters for questionnaire (female).

\section{Abbreviations}

BMI: Body mass index; KIOM: Korea Institute of Oriental Medicine; MLR: Multinomial logistic regression; SC: Sasang constitution; SCM: Sasang constitutional medicine; SE: So-Eum; SY: So-Yang; TE: Tae-Eum; TY: Tae-Yang; QSCC: Questionnaire for the Sasang constitution classification.

\section{Competing interests}

The authors hold and are currently applying for patents relating to the content of the manuscript.

\section{Authors' contributions}

$\mathrm{JHD}$ and $\mathrm{EJ}$ conceived the idea, designed the experiments, and interpreted the experimental results. BK, JSJ, and HK performed statistical analysis and interpretation of data. JYK designed the study and interpreted the experimental results. All authors contributed to manuscript preparations and approved the final manuscript.

\section{Acknowledgement}

This work was supported by the National Research Foundation of Korea (NRF) grant funded by the Korea government (MEST) (No.20110027738, 20110027739).

\section{Author details}

${ }^{1}$ Constitutional Medicine \& Diagnosis Research Group, Medical Research Division, Korea Institute of Oriental Medicine, 1672 Yuseongdae-ro, Yuseong-gu, Daejeon 305-811, Republic of Korea. ${ }^{2}$ Department of Information and Statistic, Chungnam National University, 79 Daehang-ro, Yuseong-gu, Daejeon 305-764, Republic of Korea.

Received: 18 January 2012 Accepted: 13 June 2012

Published: 4 July 2012

\section{References}

1. Song IB: An Introduction to Sasang Constitutional Medicine. Seoul: Jimoondang; 2005 .

2. Kim JY, Pham DD, Koh BH: Comparison of Sasang Constitutional Medicine, Traditional Chinese Medicine and Ayurveda. Evid Based Complement Alternat Med 2011, 2011:239659.

3. Kim JY, Pham DD: Sasang Constitutional Medicine as a Holistic Tailored Medicine. Evid Based Complement Alternat Med 2009, 6(Suppl 1):11-19.

4. Eisenberg DM, Davis RB, Ettner SL, Appel S, Wilkey S, Rompay MV, Kessler RC: Trends in Alternative Medicine Use in the United States, 1990-1997: results of a follow-up national survey. JAMA 1998, 280(18):1569-1575.
5. MacLennan AH, Wilson DH, Taylor A: Prevalence and Cost of Alternative Medicine in Australia. Lancet 1996, 347(9001):569-573.

6. Ernst E: The Role of Complementary and Alternative Medicine. BMJ 2000 321:1133-1135.

7. O'Brien KA, Abbas E, Zhang J, Guo ZX, Luo R, Bensoussan A, Komesaroff PA: Understanding the Reliability of Diagnostic Variables in a Chinese Medicine Examination. J Altern Complement Med 2009, 15(7):727-734.

8. Choi SM, Lee SH, Yoon YS, Kim JY: The Study of Data factors for SCIB (Sasang Constitution Information Bank). J Sasang Constitut Med 2005, 17(1):45-55.

9. Lee EJ, Sohn EH, Yoo JH, Kim JW, Kim KK, Kho BH, Song IB: The Study of Sasangin's Face. J Sasang Constitut Med 2005, 17(3):55-68.

10. Koo I, Kim JY, Kim MG, Kim KH: Feature Selection from a Facial Image for Distinction of Sasang Constitution. Evid Based Complement Alternat Med 2009, 6(Suppl 1):65-71.

11. Seok JH, Song JH, Kim HJ, Yoo JH, Kwak CK, Lee JH, Kho BH, Kim JW, Lee EJ: An Hardware Error Analysis of 3D Automatic Face Recognition Apparatus (3DAFRA): surface reconstruction. J Sasang Constitut Med 2007, 19(2):30-39.

12. Yun JH, Lee SK, Lee EJ, Koh BH, Song IB: Morphological Standardization Research of Head and Face on the 50's and 60's in Korean according to Sasang Constitution. J Sasang Constitut Med 2000, 12(2):123-131.

13. Pham DD, Do JH, Ku B, Lee HJ, Kim H, Kim JY: Body Mass Index and Facial Cues in Sasang Typology for Young and Elderly Persons. Evid Based Complement Alternat Med 2011, 2011:749209.

14. Huh MH, Song JM, Kim DL, Koh BH: A Study on the Morphological Diagramming of Four Constitutions. J Sasang Constitut Med 1992. 4(1):107-148.

15. Huh MH, Koh BH, Song IB: The Body Measuring Method to Classify Sasang Constitutions. J Sasang Constitut Med 2002, 14(1):51-66.

16. Kim JW, Yeo HR, Kim KK, Jeon SH, Lee MH, Lee YT: Study on the Characteristics of Body Shapes Classified by Sasang Constitution According to Age Groups. Kor J Orient Physiol Pathol 2007, 21(1):258-262.

17. Kim JW, Jeon SH, Sul YK, Kim KK, Lee EJ: A Study on the Body Shape Classified by Sasang Constitutions and Gender using Physical Measurements. J Sasang Constitut Med 2006, 18(1):54-61.

18. Shin M, Kim D: A Study on the Correlation between Sound Characteristics and Sasang Constitution by CSL. J Sasang Constitut Med 1999, 11(1):137-157.

19. Yang S, Kim D: A Study on the Correlation between Sound Spectrogram and Sasang Constitution. J Sasang Constitut Med 1996, 8(2):191-202.

20. Kim D, Park S, Gun G: An Objective Study of Sasang Constitution Diagnosis by Sound Analysis. J Sasang Constitut Med 1998, 10(1):65-80.

21. Kim DJ, Jung WK, Choi JW, Kim DR, Jeon JW: A Study on the Characteristics of the Adult Men Sound as by Sasang Constitution Analyzed with PSSC-2004. J Sasang Constitut Med 2005, 17(1):67-83.

22. Kim SH, Han DY, Youn JY, Kim DR, Jeon JW: A Study on the Characteristics of Korea Adult Women Sound as by Sasang Constitution Analysed with PSSC-2004. J Sasang Constitut Med 2005, 17(1):84-102.

23. Koh BH, Song IB: A study on the Method of Sasang Constitution Classification. J of Korean Oriental Medicine 1985, 8:146-150.

24. Kim SH, Kho BH, Song IB: A Validation Study of Questionnaire of Sasang Constitution Classification (QSCC). J Sasang Constitut Med 1993, 5(1):61-80.

25. Yoo JH, Kim JW, Kim KK, Kim JY, Koh BH, Lee EJ: Sasangin Diagnosis Questionnaire: Test of Reliability. J Altern Complement Med 2007, 13(1):111-122

26. Song KH, Yu SG, Cha S, Kim JY: Association of the apolipoprotein A5 gene $1131 \mathrm{~T}>$ C polymorphism with serum lipids in Korean subjects: Impact of Sasang constitution. Evid Based Complement Alternat Med 2012, 2012:598394.

27. Development of Diagnostic and Herbal Drug System based on Traditional Constitutional Korean Medicine. Korea: Institute of Oriental Medicine; 2007.

28. Hidden Markov Model Toolkit. http://htk.eng.cam.ac.uk.

29. Praat: doing phonetics by computer. http://www.fon.hum.uva.nl/praat/

30. Weka. http://www.cs.waikato.ac.nz/ml/weka/.

31. Jang ES, Kim MG, Baek YW, Kim YJ, Kim JY: Influence of Cold and Heat Characteristics and Health State in Sasang Constitution Diagnosis. J Sasang Constitut Med 2009, 21(3):76-88.

32. Brys G, Hubert M, Rousseeuw PJ: A Robustification of Independent Component Analysis. J Chemometrics 2005, 19:364-375. 
33. Hubert M, Vandervieren E: An Adjusted Boxplot for Skewed Distribution. Comput Stat Data Anal 2008, 52(12):5186-5201.

34. Chatterjee S, Hadi AS: Regression analysis by example. Hoboken: John Wiley and Sons; 2006

35. Agresti A: Categorical Data Analysis. Hoboken: John Wiley and Sons; 2002.

36. Stepwise Variable Selection. http://publib.boulder.ibm.com/infocenter/ spssstat/v20rOm0/index.jsp?topic=\%2Fcom.ibm.spss.statistics.help\% 2Falg_nomreg_stepwise.htm.

37. Park H, Ju J, Kim J, Kim K: A Study on clinical application of the QSCCII (Questionnaire for the Sasang Constitution Classification II). J Sasang Constitut Med 2002, 14(2):35-44.

38. Jang ES, Kim HS, Yoo JH, Kim SH, Baek YH, Lee SW: The Verification of Concordance Coefficient in Sasang Constitutional Factors by Expert. J Sasang Constitut Med 2009, 21(2):79-86.

39. Hahm TI, Hwang MW, Lee TG, Kim SB, Lee SK, Koh BH: A study on the Concordance Rate of the Diagnostic Results among Sasang Constitutional Medicine(SCM) Specialists. J Sasang Constitut Med 2006, 18(3):124-130.

doi:10.1186/1472-6882-12-85

Cite this article as: Do et al.: Development of an integrated Sasang constitution diagnosis method using face, body shape, voice, and questionnaire information. BMC Complementary and Alternative Medicine 2012 12:85

\section{Submit your next manuscript to BioMed Central and take full advantage of:}

- Convenient online submission

- Thorough peer review

- No space constraints or color figure charges

- Immediate publication on acceptance

- Inclusion in PubMed, CAS, Scopus and Google Scholar

- Research which is freely available for redistribution 